\title{
Identificação microbiológica e sensibilidade in vitro de Candida isoladas da cavidade oral de indivíduos HIV positivos
}

\author{
Microbiological identification and in vitro sensitivity of Candida \\ isolates from the oral cavity of HIV-positive individuals
}

\author{
Márcia Arias Wingeter ${ }^{1}$, Eliana Guilhermetti ${ }^{2}$, Cristiane Suemi Shinobu ${ }^{2}$, \\ Ione Takaki ${ }^{2}$ Terezinha Inez Estivalet Svidzinski ${ }^{3}$
}

\begin{abstract}
RESUMO
A candidíase orofaríngea é a infecção fúngica mais comum entre os pacientes infectados pelo vírus da imunodeficiência bumana e seu tratamento é realizado com antifúngicos tópicos ou sistêmicos, que são indicados empiricamente com base em dados clínicos. O objetivo deste estudo foi determinar a freqüência de leveduras em lavados bucais de indivíduos HIV positivos, comparando os resultados entre pacientes com diferentes condições de imunodeficiência e verificar o perfil de susceptibilidade das espécies isoladas frente aos antifúngicos visando avaliar se as opções de tratamento utilizadas na prática clínica atingem a maioria das espécies identificadas. Leveduras foram isoladas em $58 \%$ das amostras de lavado bucal coletadas e Candida albicans foi a espécie mais (93\%) freqüente. Resistência ou susceptibilidade dose dependente, frente aos antifúngicos testados foi registrada em aproximadamente $17 \%$ das amostras. A importante variabilidade de resposta sugere limitações quanto à eficácia das terapias instituídas empiricamente.
\end{abstract}

Palavras-chaves: Antifúngicos. Candidiase oral. Pacientes HIV positivos. Candida albicans.

\begin{abstract}
Oropharyngeal candidiasis is the most common fungal infection among patients infected with the human immunodeficiency virus. It can be treated with either systemic or topical antifungal agents, which are indicated empirically on the basis of clinical data. The objective of this study was to determine the frequency of yeast in mouthwashes from HIV-positive patients, compare the results between patients presenting different states of immunodeficiency, and investigate the susceptibility profile of the species isolated in relation to antifungal agents, with the aim of evaluating whether the treatments used in clinical practice are able to reach the majority of the species identified. Yeasts were isolated from $58 \%$ of the mouthwash samples collected. Candida albicans was the most (93\%) frequent species. Resistance or dose-dependent susceptibility in relation to the antifungal agent tested was registered in approximately $17 \%$ of the samples. The significant variability of responses suggests that there are limitations regarding the effectiveness of the empirical therapies instituted.
\end{abstract}

Key-words: Antifungal agents. Oral candidiasis. HIV-positive patients. Candida albicans.

Candidíase orofaríngea é a infecção fúngica mais comum entre os pacientes infectados pelo vírus da imunodeficiência humana $(\mathrm{HIV})^{2}{ }^{6}$, muitas vezes é detectada em episódios recorrentes, principalmente quando a contagem de linfócitos CD4 for baixa $^{39}$. Com a utilização dos novos anti-retrovirais na terapia HAART (highly active antiretroviral therapy) passou a observarse uma redução na ocorrência de infecções oportunistas, porém para os pacientes de diagnóstico tardio ou aqueles que não respondem adequadamente à terapêutica anti-retroviral, a candidíase orofaríngea ainda é muito freqüente ${ }^{526}$.
O tratamento e o controle da recorrência dos episódios, em lesões pouco extensas, podem ser exclusivamente tópico empregando a nistatina suspensão oral ou outras opções como o clotrimazol comprimidos para dissolver na boca e o miconazol gel. Não havendo resposta adequada ou, na presença de lesões extensas, está indicado o tratamento sistêmico, que pode ser realizado com fluconazol. Esta tem sido a droga mais utilizada devido a sua boa absorção, baixa toxicidade e disponibilidade para uso oral e endovenoso. Já o cetoconazol e itraconazol, por via oral, têm sido menos utilizado, o primeiro devido a sua

\footnotetext{
1. Serviço de Infectologia, Hospital Universitário Regional de Maringá. Maringá, PR. 2. Laboratório de Ensino e Pesquisa em Análises Clínicas, Universidade Estadual de Maringá, Maringá, PR. 3. Disciplina de Micologia Médica, Universidade Estadual de Maringá, Maringá, PR.

Endereço para correspondência: Profa. Terezinha Inez Estivalet Svidzinski. R. Júlio Favoretto 35, Vila Esperança, 87020-600 Maringá, PR.

Tel: 5544 3261-4809; Fax: 5544 3261-4959

e-mail: tiesvidzinski@uem.br

Recebido para publicação em 26/7/2007

Aceito em 26/4/2007
} 
toxicidade hepática e pela contra-indicação em associações com diversos medicamentos, inclusive com anti-retrovirais e o itraconazol, apesar da baixa toxicidade apresenta diversas interações medicamentosas desfavoráveis. A anfotericina B, para uso endovenoso, está estritamente reservada aos casos graves, devido às freqüientes reações adversas ${ }^{25}$. Novas drogas antifúngicas como a caspofungina estão sendo empregadas em protocolos recentes ${ }^{118}$, entretanto ainda não são utilizadas na prática clínica devido ao elevado custo.

0 tratamento da candidíase oral em geral é instituído empiricamente, porém é importante lembrar que essa infecção pode apresentar-se com sintomas e aparência macroscópica bastante variáveis ${ }^{25}$. Esse polimorfismo exigiria do clínico a necessidade de adequação da terapêutica, contudo o tratamento medicamentoso utilizado na candidíase orofaríngea, em pacientes infectados pelo HIV, é instituído empiricamente e muitas vezes por tempo prolongado. Este fato contribui para o aparecimento de espécies de leveduras resistentes ${ }^{19}$. Este fato, aliado à pouca disponibilidade de novos produtos desenvolvidos pela indústria farmacêutica tem motivado vários estudos sobre a susceptibilidade in vitro dos fungos agentes de infecções humanas, principalmente em imunocomprometidos ${ }^{120}$.

O objetivo deste estudo foi determinar a frequiência de leveduras em lavados bucais de indivíduos HIV positivos, comparando os resultados entre pacientes com diferentes condições de imunodeficiência e verificar o perfil de susceptibilidade das espécies isoladas frente aos antifúngicos, com vistas em avaliar se as opções de tratamento utilizadas na prática clínica atingem a maioria das espécies identificadas.

\section{MATERIAL E MÉTODOS}

No período de julho a dezembro de 2003, foram coletadas amostras de lavado bucal de pacientes com infecção pelo HIV ou portadores de AIDS, que compareceram ao Laboratório de Ensino e Pesquisa em Análises Clínicas (LEPAC) da Universidade Estadual de Maringá para a coleta periódica dos exames para o acompanhamento da doença.

O LEPAC é o laboratório público de referência que atende pelo Sistema Único de Saúde (SUS) onde são realizados os exames para diagnóstico e controle de tratamento da AIDS em Maringá.

Os pacientes incluídos na pesquisa tinham o diagnóstico confirmado de infecção pelo HIV ou eram doentes de AIDS, conforme os critérios da vigilância epidemiológica de $1998^{3}$. Todos tinham idade superior a 18 anos e estavam sendo acompanhados no Serviço de Atendimento Especializado para pacientes infectados pelo HIV da 15 $5^{\text {a }}$ Regional de Saúde do Paraná e do Ambulatório de Infectologia do Hospital Universitário Regional de Maringá (HUM). Foram excluídos do trabalho os indivíduos em vigência de outras terapias imunossupressoras.

Os pacientes foram esclarecidos e convidados a participar voluntariamente do estudo e assinaram o termo de consentimento aprovado pelo Comitê Permanente de Ética em Pesquisa Envolvendo Seres Humanos da Universidade Estadual de
Maringá (COPEP- UEM). Os dados clínicos foram obtidos a partir de consulta médica e revisão do prontuário.

Foi obtida uma amostra biológica de cada paciente através de bochecho, por 30 segundos, com 10ml de solução fisiológica esterilizada. 0 líquido resultante do bochecho foi recolhido e, após ser centrifugado por 30 segundos a 2.000rpm, foi ressuspenso em $1 \mathrm{ml}$ de salina tamponada (PBS pH 7,2), e alíquotas de 10 $\mathrm{\mu l}$ foram cultivadas em placas de Petri contendo o meio seletivo diferencial CHROMágar ${ }^{\circledR}$ Candida (CHROMagar Company, Paris, França) e incubadas a $25^{\circ} \mathrm{C}$ por até cinco dias. A seguir as colônias individuais de cada amostra foram identificadas ao nível de gênero e espécie baseado na produção de tubo germinativo, micromorfologia em agar fubá com tween 80, além das provas bioquímicas assimilação e fermentação de carboidratos de acordo com Larone ${ }^{11}$, complementado por Kurtzman \& $\mathrm{Fe}^{10}{ }^{10}$. A presença de Candida dubliniensis foi descartada pela incubação em ágar salgado a $45^{\circ} \mathrm{C}$.

Testes de susceptibilidade aos antifúngicos. Os testes de microdiluição em caldo para determinação do perfil de susceptibilidade das leveduras aos antifúngicos clássicos foram realizados de acordo com a metodologia padronizada pelo National Committee for Clinical Laboratory Standards publicadas no documento $\mathrm{M}-27 \mathrm{~A}^{16}$.

Os antifúngicos testados foram fluconazol (Galena Química e Farmacêutica), itraconazol (Janssen Pharmaceutica), cetoconazol (Janssen Pharmaceutica), nistatina (Sigma N 3503, USA) e anfotericina B (Fungizon ${ }^{\circledR}$, Bristol-Myers Squibb Brasil). Foram utilizadas concentrações variando de 0,125 a $64 \mu \mathrm{g} / \mathrm{ml}$ para 0 fluconazol e nistatina e de 0,03 a $16 \mu \mathrm{g} / \mathrm{ml}$ para itraconazol, cetoconazol e anfotericina $\mathrm{B}$.

Ao meio de cultura RPMI-1640 (Sigma) foi adicionado tampão (MOPS) e glicose e ajustado o pH para 7,0 com ácido clorídrico concentrado P.A. (Merck). Alíquotas de $100 \mu \mathrm{l}$ de dez concentrações de cada antifúngico foram dispensadas seqüencialmente nas colunas numeradas de 1 a 10 de cada placa de microtitulação de 96 poços (Nunclon, Delta, Nunc A/S, Roskilde, Denmark).

A concentração do inóculo foi ajustada em 90\% de transmitância em 530nm spectrofotômetro (Baush \& Lomb) de modo que 0 volume de $100 \mu$ l dessa suspensão, adicionado aos antifúngicos já distribuídos na placa, continha entre 0,5 e 2,5 x $10^{3} \mathrm{UFC} / \mathrm{ml}$. $\mathrm{Na}$ coluna onze, foi distribuído o inóculo aferido sobre $100 \mu \mathrm{l}$ do meio de cultura isento de drogas para proporcionar $100 \%$ de crescimento da levedura testada (controle positivo) e na coluna 12 foram dispensados 200 $\mu$ l de meio RPMI-1640 (controle negativo). As placas com as drogas e inóculo foram incubadas, em câmara úmida, a $35^{\circ} \mathrm{C}$ por 48 horas. A leitura do teste foi feita através de comparação visual, por reflexão em espelho.

A atividade das drogas antifúngicas e a reprodutibilidade dos resultados foram asseguradas pela inclusão do isolado de referência Candida parapsilosis ATCC 22019 em todos os ensaios realizados.

0 critério de leitura considerou que a concentração inibitória mínima (CIM) seria aquela capaz de inibir 50\% do crescimento para os azólicos e 90\% para os poliênicos. 
Na Tabela 1 é apresentada a interpretação dos valores de CIM considerados para os cinco antifúngicos testados. Para fluconazol, itraconazol e anfotericina $B$ foi considerado o documento NCCLS - M27-A, já para cetoconazol e nistatina utilizou-se critérios estabelecidos pelo laboratório já considerados em estudos anteriores ${ }^{17}$.

Tabela 1 - Interpretação dos valores de concentração inibitória mínima dos cinco antifúngicos testados.

\begin{tabular}{llcc}
\hline \multirow{2}{*}{ Antifúngico } & \multicolumn{3}{c}{ Concentração inibitória mínima $(\mu \mathrm{g} / \mathrm{ml})$} \\
\cline { 2 - 4 } & sensível & $\begin{array}{c}\text { intermediário ou } \\
\text { sensibilidade } \\
\text { dose-dependente }\end{array}$ \\
\hline Fluconazol & $\leq 8,0$ & 16 a 32 & $\geq 64,0$ \\
Itraconazol & $\leq 0,125$ & 0,25 a 0,5 & $\geq 1,0$ \\
Cetoconazol & $\leq 0,125$ & 0,25 a 0,5 & $\geq 1,0$ \\
Nistatina & $\leq 4,0$ & 8 a 32 & $\geq 64,0$ \\
Anfotericina B & $<1,0$ & - & $>2,0$ \\
\hline
\end{tabular}

Análise estatística. Foi utilizado o Teste Exato de Fisher como teste de homogeneidade para verificar se existe diferença significativa ao nível de 5\% entre a susceptibilidade das leveduras isoladas de pacientes com $\mathrm{CD} 4$ abaixo ou acima de $200 \mathrm{cel} / \mathrm{mm}^{3}$.

\section{RESULTADOS}

Dos 100 pacientes infectados pelo HIV, 58 apresentaram culturas positivas para Candida spp. Destes, 29 eram homens e 29 mulheres e a idade variou entre 19 e 69 anos, com média de 39 anos. A contagem de linfócitos CD4 variou entre 2 e 1.128 cel/ $\mathrm{mm}^{3}$, em média de $364 \mathrm{cel} / \mathrm{mm}^{3}, 12$ (21\%) pacientes tinham CD4 $<200 \mathrm{cel} / \mathrm{mm}^{3}$. A carga viral superior a 100.000 cópias $/ \mathrm{ml}$, foi verificada em 13 (22\%) pacientes. Doze (21\%) pacientes apresentaram pelo menos um episódio prévio de candidíase oral e 13 (22\%) já haviam utilizado antifúngicos, sendo que cinco deles utilizaram mais de um produto. Os antifúngicos utilizados foram: anfotericina $B$ em 2 pacientes, nistatina em 3, cetoconazol em 4 e fluconazol em 11 pacientes. Cinquienta e um pacientes estavam fazendo uso de terapia anti-retroviral, sendo que 47 (81\%) há mais de um ano.

Candida albicans foi a espécie isolada na maioria (93\%) dos pacientes, somente em quatro (7\%) deles foram isoladas outras espécies de leveduras Candida tropicalis (2) e Candida glabrata (2).
Tabela 2 - Perfil de susceptibilidade in vitro de isolados de Candida sp aos antifúngicos.

\begin{tabular}{lcrrrr}
\hline \multirow{2}{*}{ Organismos } & \multicolumn{5}{c}{ Variação da concentração inibitória mínima $(\mu \mathrm{g} / \mathrm{ml})$} \\
\cline { 2 - 7 } Candida albicans & $0,03-16$ & $2-64$ & $0,03-16$ & $0,125-64$ & $0,03-16$ \\
Canfotericina B & nistatina & cetoconazol & fluconazol & itraconazol \\
\hline
\end{tabular}

A Tabela 2 apresenta a variação das CIM de cada antifúngico testado. 0 padrão de susceptibilidade, analisando todos os isolados, mostrou amplo espectro de variação frente aos antifúngicos testados e, apesar do pequeno número, as amostras de Candida não albicans apresentaram CIM mais elevadas.

$\mathrm{Na}$ Tabela 3 é possível observar a distribuição dos isolados conforme a concentração de cada droga. Para a nistatina, 95\% dos isolados foram inibidos por até $4 \mu \mathrm{g} / \mathrm{ml}$, enquanto $\mathrm{CIM} \leq 8 \mu \mathrm{g} / \mathrm{ml}$ de fluconazol foi suficiente para inibir $86 \%$ dos isolados.

Durante a realização dos testes, ocorreu a perda de duas amostras, que não apresentaram crescimento, uma para o teste com anfotericina B e outra para o teste com itraconazol, portanto para estes antifúngicos foram consideradas 57 amostras (Tabela 4).

Para o cetoconazol e itraconazol, a variação das CIM foi maior, mesmo assim $72 \%$ dos isolados apresentaram CIM $<1 \mu \mathrm{g} / \mathrm{ml}$ para o cetoconazol, enquanto $77 \%$ deles foram susceptíveis ao itraconazol em $\mathrm{CIM}<1 \mu \mathrm{g} / \mathrm{ml}$. Dois dos 57 isolados testados demonstraram CIM $>2 \mu \mathrm{g} / \mathrm{ml}$ para a anfotericina $\mathrm{B}$ (Tabelas $3 \mathrm{e} 4$ ).

De forma geral, a sensibilidade foi alta, mais de $60 \%$ dos isolados foram sensiveis a todos os antifúngicos testados conforme demonstra a Tabela 4, sendo que para o cetoconazol foi observado maior número de isolados resistentes (28\%) e de susceptibilidade dose-dependente (5\%).

A análise estatística, não mostrou diferença significativa entre os grupos de pacientes com CD 4 abaixo ou acima de $200 \mathrm{cel} / \mathrm{mm}$, quando avaliado a susceptibilidade ao cetoconazol, fluconazol e itraconazol. Para nistatina e anfofericina $\mathrm{B}$, a análise estatística foi desnecessária, visto que os resultados dos dois grupos eram muito semelhantes. Entre os pacientes com CD4 abaixo de 200 $\mathrm{cel} / \mathrm{mm}^{3}$, todos os isolados foram sensíveis a anfotericina B e nistatina, enquanto que entre os pacientes com $\mathrm{CD} 4$ acima de $200 \mathrm{cel} / \mathrm{mm}^{3}$ a sensibilidade foi $95,2 \%$ para a anfotericina B e $96 \%$ para a nistatina.

Tabela 3 - Distribuição dos isolados de Candida sp conforme a concentração inibitória mínima de cada um dos cinco antifúngicos analisados.

\begin{tabular}{|c|c|c|c|c|c|c|c|c|c|c|c|c|}
\hline & \multicolumn{10}{|c|}{ Número de isolados inibidos nas concentrações $(\mu \mathrm{g} / \mathrm{ml})$ de: } & \multicolumn{2}{|c|}{$\mathrm{CIM}$} \\
\hline & 0,125 & 0,25 & 0,5 & 1 & 2 & 4 & 8 & 16 & 32 & 64 & $\mathrm{CIM}_{50}$ & $\mathrm{CIM}_{90}$ \\
\hline Nistatina & 0 & 0 & 0 & 0 & 6 & 49 & 2 & 0 & 0 & 1 & 4 & 4 \\
\hline \multirow[t]{2}{*}{ Fluconazol } & 1 & 4 & 15 & 16 & 10 & 3 & 1 & 0 & 2 & 6 & 1 & 64 \\
\hline & 0,03 & 0,06 & 0,125 & 0,25 & 0,5 & 1 & 2 & 4 & 8 & 16 & $\mathrm{CIM}_{50}$ & $\mathrm{CIM}_{90}$ \\
\hline Cetoconazol & 30 & 1 & 8 & 2 & 1 & 3 & 5 & 2 & 5 & 1 & 0,03 & 8 \\
\hline Anfotericina B & 9 & 39 & 7 & 0 & 0 & 0 & 0 & 1 & 0 & 1 & 0,06 & 0,125 \\
\hline Itraconazol & 10 & 23 & 8 & 1 & 2 & 3 & 1 & 1 & 2 & 6 & 0,06 & 16 \\
\hline
\end{tabular}

CIM: concentração inibitória mínima 
Tabela 4 - Número e porcentagem de isolados de Candida sp considerados sensiveis, susceptibilidade dose-dependente ou intermediários e resistentes.

\begin{tabular}{|c|c|c|c|c|c|c|}
\hline & \multicolumn{2}{|c|}{ Sensível } & \multicolumn{2}{|c|}{ Intermediário (SDD) } & \multicolumn{2}{|c|}{ Resistente } \\
\hline & $\mathrm{n}^{0}$ & $\%$ & $\mathrm{n}^{0}$ & $\%$ & $\mathrm{n}^{0}$ & $\%$ \\
\hline Fluconazol $(\mathrm{n}=58)$ & 50 & 86 & 2 & 4 & 6 & 10 \\
\hline Itraconazol $(\mathrm{n}=57)$ & 41 & 72 & 3 & 5 & 13 & 23 \\
\hline Cetoconazol $(\mathrm{n}=58)$ & 39 & 67 & 3 & 5 & 16 & 28 \\
\hline Nistatina $(\mathrm{n}=58)$ & 55 & 95 & 2 & 3 & 1 & 2 \\
\hline Anfotericina B $(n=57)$ & 55 & 96 & 0 & & 2 & 4 \\
\hline
\end{tabular}

SDD: susceptibilidade dose-dependente ou intermediária

\section{DISCUSSÃO}

No presente estudo, foram coletadas amostras de lavado bucal de 100 pacientes infectados pelo HIV. Destes, 58 apresentaram culturas positivas para Candida sp, dos quais $20 \%$ apresentavam sintomas ou sinais de candidíase oral. A taxa de colonização por leveduras de 58\% destes pacientes HIV positivos foi similar à encontrada em outros estudos ${ }^{213}$. Não é considerada elevada para essa população, visto que 60 a $80 \%$ desses pacientes desenvolvem uma ou mais infecções fúngicas durante sua doença, sendo a candidíase de orofaringe a mais freqüente ${ }^{21}$. Entre indivíduos normais a taxa média de colonização é de $34,4 \%{ }^{2}$.

A média de idade dos pacientes com culturas positivas foi 39 anos, um pouco acima da faixa de 30 a 34 anos que apresenta a maior incidência de AIDS no Brasil ${ }^{15}$. Apesar do número de amostras positivas provenientes de homens e mulheres ser equivalente, a frequiência de diagnósticos para AIDS entre os gêneros, na região avaliada, segue a tendência nacional segundo o Ministério da Saúde ${ }^{14}$ (1,5 homens para cada mulher). A maior proporção de mulheres no estudo talvez possa ser justificada pela característica feminina de maior assiduidade na realização dos exames de rotina e dedicação aos assuntos relacionados à saúde bem como na disposição para participar da pesquisa.

CD 4 médio de $364 \mathrm{cel} / \mathrm{mm}^{3}$ e carga viral baixa, na maioria dos pacientes, indicam que nessa população a imunodeficiência estava sob controle, fato possivelmente relacionado com a aderência ao tratamento, pois 51 pacientes estavam em uso da terapia antiretroviral, sendo que 47 (81\%) o utilizavam há mais de 1 ano. Apesar disso, a projeção em relação à população estudo merece atenção: a situação clínica favorável, portanto maior expectativa de vida e a importante frequiência de colonização por Candida sp, são duas características particulares que, se tomadas em conjunto, sugerem alta probabilidade de ocorrência de candidíase oral no decorrer do acompanhamento desses pacientes.

A Tabela 2 mostra que há grande variação quanto à capacidade de ação de cada droga antifúngica conforme o microrganismo isolado. Tendo em vista que a escolha da terapêutica é empírica e não é realizado rotineiramente nenhum tipo de teste para verificar a eficácia das drogas a serem utilizadas. Estes resultados sugerem diferentes respostas clínicas às opções de tratamento utilizadas.

A variação no padrão de susceptibilidade dos isolados de Candida sp aos antifúngicos é confirmado pela Tabela 3. Enquanto $95 \%$ dos isolados apresentaram $\mathrm{CIM} \leq 4 \mu \mathrm{g} / \mathrm{ml}$ à nistatina mostrando comportamento uniforme, em relação ao fluconazol, embora $86 \%$ dos isolados testados apresentaram $\mathrm{CIM} \leq 8 \mu \mathrm{g} / \mathrm{ml}$, a distribuição das leveduras em relação às diferentes concentrações da droga foi muito mais ampla e variável. Apesar da tendência à susceptibilidade, para estas duas opções terapêuticas mais utilizadas na prática, as variações observadas nas Tabelas 2 e 3 sugerem a possibilidade de falha clínica com o tratamento empírico.

Neste estudo, $\mathrm{a} \mathrm{CIM}_{90}$ das leveduras ao fluconazol foi $64 \mu \mathrm{g} / \mathrm{ml}$ (Tabela 3) e 14\% das amostras foram resistentes ou S-DD a esse antifúngico (Tabela 4), índice superior ao encontrado por outros autores $^{402223}$.

Em recente estudo multicêntrico realizado no Brasil, a freqüência de isolados resistentes e com S-DD aos azólicos foi de $11 \%$, sendo que na maioria destes casos os pacientes apresentavam a contagem de linfócitos CD4 abaixo de 100 cels/ $\mathrm{mm}^{3}$ e tinham história prévia de candidíase de orofaringe com tratamento antifúngico ${ }^{13}$.

Para o cetoconazol e itraconazol, foi observada uma variação maior de susceptibilidade entre as amostras testadas, $72 \%$ dos isolados apresentam $\mathrm{CIM}<1 \mu \mathrm{g} / \mathrm{ml}$ para o cetoconazol, enquanto $77 \%$ foram susceptíveis ao itraconazol em CIM $<1 \mu \mathrm{g} / \mathrm{ml}$ (Tabela 3).

Ouso deitraconazol profilático empacientes com AIDSfoi associado à redução da susceptibilidade ao fluconazol para espécies isoladas de Caindida albicans em episódios de candidíase oral recorrente ${ }^{7}$.

Apesar dos mais de 50 anos de uso dos polienos, a resistência a anfotericina B é rara, porém, neste estudo dois dos 57 isolados testados demonstraram CIM $>2 \mu \mathrm{g} / \mathrm{ml}$ para a anfotericina $\mathrm{B}$, caracterizando resistência.

Neste estudo, não houve diferença estatística, quanto ao perfil de susceptibilidade aos antifúngicos, entre as leveduras isoladas de pacientes com contagens de CD4 superiores ou inferiores a $200 \mathrm{cel} / \mathrm{mm}^{3}$, o que está de acordo com outros autores ${ }^{512}{ }^{13}$. Por outro lado a importante variabilidade encontrada entre os isolados sugere maior valorização das características relacionadas ao agente. É possível que em curto período de tempo os protocolos de acompanhamento dos pacientes HIV positivos incluam a confirmação laboratorial da espécie e perfil de susceptibilidade das leveduras isoladas. Uma rotina desse tipo talvez estimulasse a indústria farmacêutica a diversificar o arsenal terapêutico para a candidíase oral e o oferecimento de novas opções de drogas antifúngicas para uso tópico sobre mucosas, tendo em vista a forte ingestão de medicamentos orais por essa população.

\section{REFERÊNCIAS}

1. Bachmann SP, Patterson TF, Ribot JLL. In vitro activity of caspofungin (MK-0991) against Candida albicans clinical isolates displaying different mechanisms of azole resistance. Journal Clinical Microbiology 40:2228-2230, 2002.

2. Campisi G, Pizzo G, Milici ME, Mancuso S, Margiotta V. Candidal carriage in the oral cavity of human immunodeficiency virus-infected subjects. Oral Medicine $93: 281-286,2002$.

3. Campos J, Del Romero J, Castilha J, Garcia S, Rodriguez C, Bascones A. Oral candidiasis as a clinical marker related to viral load, CD4 lymphocyte count 
and CD4 lynphocyte percentage in HIV-infected patients. Journal Oral Pathology Medicine 31:5-10, 2002

4. Colombo AL, Matta D, Almeida LP, Rosas R. Fluconazole susceptibility of brazilian Candida isolates assessed by a disk diffusion method. The Brazilian Journal of Infectious Diseases 6:118-123, 2002

5. Eyeson JD, Tenant-Flowers M, Cooper DJ, Johnson NW, Warnakulasuriya KAS. Oral manifestations of an HIV positive cohort in the era of highly active anti-retroviral therapy (HAART) in South London. Journal Oral Pathology Medicine 31:169-174, 2002.

6. Fidel Jr PL. Immunity to Candida. Oral Diseases 8:69-75, 2002

7. Goldman M, Cloud GA, Smedema M, Lemonte A, Connolly P, Mckinsey D, Kauffman CA, Moskovitz B, Wheat $\mathrm{J}$ and members of the National Institute of Allergy and Infectious Diseases Mycoses Study Group. Does long-term itraconazole prophylaxis result in vitro azole resistance in mucosal Candida albicans isolates from persons which advanced human immunodeficiency virus infection? Antimicrobial Agents Chemotherapy 44:1585-1587, 2000.

8. Greenspan D, Gange SJ, Phelan JA, Navazesh M, Alves MEAF, MacPhail LA, Mulligan R, Greenspan JS. Incidence of oral lesions in HIV-1-infected women: Reduction a with HAART. Journal Dental Research 83:145-150, 2004.

9. Gupta E, Vajpayee M, Xess I, Seth P. Oral candidiasis in HIV-infected patients and its relation to $\mathrm{CD} 4$ + counts: an adjunct clinical marker of HIV disease progression. Tropical Doctor 36:127, 2006.

10. Kurtzman CP, Fell JW. The Yeast. A taxonomic study. Elsevier, Amsterdam, 1998.

11. Larone DH. Medically important fungi. A guide to identification. AMS Press, Washington, 1995.

12. Melo NR, Taguchi H, Jorge J, Pedro RJ, Almeida OP, Fukushima K, Nishimura K, Mivaji M. Oral Candida flora from Brazilian human immunodeficiency virusinfected patientes in the highly active antiretroviral therapy era. Memórias do Instituto Oswaldo Cruz 99:425-431, 2004.

13. Milan EP. Avaliação fenotípica e genotípica de leveduras isoladas da cavidade oral de pacientes com AIDS. Dissertação de Doutorado, Universidade Federal de São Paulo, São Paulo, SP, 2001.

14. Ministério da Saúde. Secretaria de Vigilância em Saúde. Programa Nacional de DST e AIDS. Critérios de definição de casos de AIDS em adultos e crianças. Ministério da Saúde, Brasília, 2003.

15. Ministério da Saúde. Secretaria de Vigilância em Saúde. Programa Nacional de DST e AIDS. Boletim Epidemiológico AIDS - DST. Janeiro a Junho de 20041. Ministério da Saúde, Brasília, 2005.
16. National Committee for Laboratory Standards NCCLS. Reference method for broth dilution antifungal susceptibility testing of yeast. Approved Standard. NCCLS M27A. 17:1-29, 1997 .

17. Pádua RAF, Guilhermetti E, Svidzinski TIE. In vitro activity of antifungal agents on yeasts isolated from vaginal secretion. Acta Scientiarum. Health Sciences 25:51-54, 2003.

18. Pfaller MA, Messer SA, Boyken L, Rice C, Tendolkar S, Hollis RJ, Diekema DJ. Caspofungin activity against clinical isolates of fluconazole-resistant Candida. Journal of Clinical Microbiology 41:5729-5731, 2003.

19. Powderly WG, Robinson K, Keath EJ. Molecular epidemiology of recurrent oral candidiasis in human immunodeficiency virus-positive patients: evidence for two patterns of recurrence. Journal of Infectious Diseases 168:463-466, 1993.

20. Rex JH, Rinald MG, Pfaller MA. Resistance of Candida species to fluconazole. Antimicrobial Agents and Chemotherapy 39:1-8, 1995.

21. Rex JH, Walsh TJ, Sobel JD, Filler SG Pappas PG, Dismukes WE, Edwards JE Pratice Guidelines for the Treatment of Candidiasis. Clinical Infectious Diseases 30:662-678, 2000

22. Sánchez-Vargas LO, Ortiz-López NG, Villar M, Moragues MD, Aguirre JM, Cashat-Cruz M, Lopez-Ribot JL, Gaitán-Cepeda LA, Quindós G. Point prevalence, microbiology and susceptibility patterns of oral Candida isolates colonizing or infecting Mexican HIV/AIDS patients and healthy persons. Revista Iberoamamericana de Micología 22:83-92, 2005.

23. Sanglard D, Kuchler K, Ischer F, Pagani JL, Monod M. Mechanisms of resistance to azole antifungal agents in Candida albicans isolates from AIDS patients involve specific multidrug transporters. Antimicrobial Agents Chemotherapy 39:2378-2386, 1995

24. Sant'Ana PL, Milan EP, Martinez R, Telles FQ, Ferreira MS, Alcântara MTC, Carvalho MT, Colombo AL. Multicenter Brazilian study of oral Candida species isolated from AIDS patients. Memórias do Instituto Oswaldo Cruz 97:253-257, 2002

25. Schechter M, Rachid M. Manual de HIV/AIDS. Revinter, Rio de Janeiro, 2004

26. Telles FQ, Silva N, Carvalho MM, Alcântara AP, Matta D, Barberino MG, Bartezak S, Colombo AL. Evaluation of efficacy and safety of itraconazole oral solution for the treatment of oropharyngeal candidiasis in AIDS patients. The Brazilian Journal of Infectious Disease 5:60-66, 2002. 